\title{
Implications of expanding indications for drug treatment to prevent fracture in older men in United States: cross sectional and longitudinal analysis of prospective cohort study
}

Kristine E Ensrud professor ${ }^{1}$ core investigator $^{2}$, Brent $\mathrm{C}$ Taylor associate professor ${ }^{1}$ core investigator $^{2}$, Katherine W Peters statistician ${ }^{3}$, Margaret L Gourlay assistant professor ${ }^{4}$, Meghan G Donaldson research associate ${ }^{5}$, William D Leslie professor ${ }^{6}$, Terri L Blackwell statistician ${ }^{3}$, Howard A Fink associate professor ${ }^{1}$ core investigator $^{2}$, Eric S Orwoll professor ${ }^{7}$, John Schousboe rheumatologist $^{8}$ assistant professor ${ }^{9}$, for the Osteoporotic Fractures in Men (MrOS) Study Group

${ }^{1}$ Department of Medicine and Division of Epidemiology and Community Health, University of Minnesota, Minneapolis, MN, USA; ${ }^{2}$ Center for Chronic Disease Outcomes Research, VA Health Care System, Minneapolis, MN, USA; ${ }^{3}$ California Pacific Medical Center Research Institute, San Francisco, CA, USA; ${ }^{4}$ Department of Family Medicine, University of North Carolina, Chapel Hill, NC, USA; ${ }^{5}$ Centre for Clinical Epidemiology and Evaluation, University of British Columbia, Vancouver, BC, Canada; ${ }^{6}$ Department of Medicine, University of Manitoba, Winnipeg, MB, Canada; ${ }^{7}$ Bone and Mineral Unit, Oregon Health and Science University, Portland, OR, USA; ${ }^{8}$ Park Nicollet Clinic, St Louis Park, MN, USA; ${ }^{9}$ Division of Health Policy and Management, University of Minnesota, Minneapolis, MN, USA

\begin{abstract}
Objectives To quantify incremental effects of applying different criteria to identify men who are candidates for drug treatment to prevent fracture and to examine the extent to which fracture probabilities vary across distinct categories of men defined by these criteria.

Design Cross sectional and longitudinal analysis of a prospective cohort study.

Setting Multicenter Osteoporotic Fractures in Men (MrOS) study in the United States.

Participants 5880 untreated community dwelling men aged 65 years or over classified into four distinct groups: osteoporosis by World Health Organization criteria alone; osteoporosis by National Osteoporosis Foundation (NOF) but not WHO criteria; no osteoporosis but at high fracture risk (at or above NOF derived FRAX intervention thresholds recommended for US); and no osteoporosis and at low fracture risk (below NOF derived FRAX intervention thresholds recommended for US).
\end{abstract}

Main outcome measures Proportion of men identified for drug treatment; predicted 10 year probabilities of hip and major osteoporotic fracture calculated using FRAX algorithm with femoral neck bone mineral density; observed 10 year probabilities for confirmed incident hip and major osteoporotic (hip, clinical vertebral, wrist, or humerus) fracture events calculated using cumulative incidence estimation, accounting for competing risk of mortality.
Results 130 (2.2\%) men were identified as having osteoporosis by using the WHO definition, and an additional 422 were identified by applying the NOF definition (total osteoporosis prevalence 9.4\%). Application of NOF derived FRAX intervention thresholds led to 936 (15.9\%) additional men without osteoporosis being identified as at high fracture risk, raising the total prevalence of men potentially eligible for drug treatment to $25.3 \%$. Observed 10 year hip fracture probabilities were $20.6 \%$ for men with osteoporosis by WHO criteria alone, $6.8 \%$ for men with osteoporosis by NOF (but not WHO) criteria, $6.4 \%$ for men without osteoporosis but classified as at high fracture risk, and $1.5 \%$ for men without osteoporosis and classified as at low fracture risk. A similar pattern was noted in observed fracture probabilities for major osteoporotic fracture. Among men with osteoporosis by WHO criteria, observed fracture probabilities were greater than FRAX predicted probabilities $(20.6 \%$ v $9.5 \%$ for hip fracture and $30.0 \% \vee 17.4 \%$ for major osteoporotic fracture).

Conclusions and relevance Choice of definition of osteoporosis and use of NOF derived FRAX intervention thresholds have major effects on the proportion of older men identified as warranting drug treatment to prevent fracture. Among men identified with osteoporosis by WHO criteria, who comprised $2 \%$ of the study population, actual observed fracture probabilities during 10 years of follow-up were highest and exceeded FRAX predicted fracture probabilities. On the basis of findings from randomized trials in women, these men are most likely to benefit from treatment. Expanding indications for treatment beyond this small group has uncertain value owing to lower observed fracture probabilities 
and uncertain benefits of treatment among men not selected on the basis of WHO criteria.

\section{Introduction}

Older men experience $29 \%$ of the fractures among adults aged 50 years and older in the United States and account for $25 \%$ of fracture related costs. ${ }^{1}$ Many of these fractures occur at sites associated with low bone mineral density, increase in incidence after age 50 years, and are considered to be related to osteoporosis. ${ }^{2}$ However, the best strategy to identify men who are candidates for drug treatment to prevent fracture is uncertain, in part because the cut-off value for bone mineral density to identify osteoporosis is less well defined in men than in women. ${ }^{4}$ The diagnosis of osteoporosis in both men and women endorsed by the World Health Organization is based on the cut-off value of femoral neck bone mineral density used in women ( $\mathrm{T}$ score -2.5 or lower at the femoral neck, calculated using the young white female normal reference database).$^{56}$ In contrast, the diagnosis of osteoporosis in men endorsed by the National Osteoporosis Foundation (NOF) ${ }^{78}$ and adopted by some, ${ }^{9}$ but not all, ${ }^{10}$ US professional societies is defined as a T score of -2.5 or lower at the femoral neck, total hip, or lumbar spine, calculated using the young white male normal reference base. Use of the NOF's definition increases the apparent prevalence of osteoporosis in men, but controversy exists regarding whether this effect is modest or substantial. ${ }^{11}{ }^{12}$

Randomized trials in men have shown that drug treatment increases bone mineral density, ${ }^{13-17}$ but the efficacy of drugs in preventing clinical fracture events (that is, symptomatic fractures coming to medical attention) in men is unknown. The efficacy of drug treatment in reducing the risk of clinical fractures has been demonstrated only in postmenopausal women with osteoporosis defined by a female specific bone mineral density $\mathrm{T}$ score of -2.5 or lower or with existing radiographic vertebral fractures, ${ }^{18}$ with the exception of one randomized trial of zoledronic acid in patients with recent hip fracture $(75 \%$ of whom were women, $42 \%$ with osteoporosis by WHO criteria) that reported efficacy of treatment in reducing future fracture events. ${ }^{19}$ Although osteoporosis by WHO criteria identifies postmenopausal women who benefit from drug treatment to lower the risk of clinical fractures, many postmenopausal women and older men with bone mineral density $\mathrm{T}$ scores above -2.5 will subsequently experience clinical fracture events. ${ }^{20}$ This common occurrence has prompted efforts to derive estimates of the absolute risk of fracture for use in making decisions about whether to recommend starting drug treatment in men, as well as women.

The FRAX algorithm is a publicly available web based tool that uses clinical risk factors and country specific data, with or without consideration of femoral neck bone mineral density measurement, to estimate an individual patient's 10 year probabilities of hip and major osteoporotic fractures (defined by FRAX as clinical vertebral, forearm, hip, or proximal humerus fracture). ${ }^{21-23}$ The development of the FRAX tool has been advocated by several organizations, ${ }^{7-9}{ }^{24}$ supported in part by funding from the pharmaceutical industry, ${ }^{25-27}$ which have advocated the use of FRAX in clinical decision making to identify high risk candidates for drug treatment to prevent fracture. This has promoted a shift in treatment approach in many practice settings from one based primarily on measurement of bone mineral density to an approach based on absolute risk of fracture.

Use of FRAX in clinical practice demands a consideration of the probability of fracture at which to intervene.$^{28}$ On the basis of a cost effectiveness analysis, ${ }^{29}$ the NOF in 2010 recommended
FRAX intervention thresholds of 3\% for hip fracture and 20\% for major osteoporotic fracture as cut points for starting drug treatment in US adults aged 50 years and older. However, a critical unproved assumption in this analysis is that drug treatment reduces risk of clinical fractures by $35 \%$ regardless of bone mineral density or the presence of existing vertebral fractures. Despite the controversies in identifying osteoporosis in men and lack of data on the efficacy of treatment in preventing clinical fracture events in populations other than postmenopausal women with osteoporosis as defined by WHO criteria or with existing vertebral fractures, current US treatment guidelines recommend starting drug treatment to prevent fracture in men with a history of hip or vertebral fracture, men with male specific bone mineral density $\mathrm{T}$ scores at or below -2.5 , and men with a male specific bone mineral density $\mathrm{T}$ score between -1.0 and -2.5 who are at or above NOF derived FRAX intervention thresholds.

We used data collected in 5880 untreated, community dwelling US men aged 65 years or above enrolled in the prospective Osteoporotic Fractures in Men (MrOS) study to determine the incremental effects of broadening the definition of osteoporosis and use of NOF derived FRAX intervention thresholds on the proportion of older men identified as candidates for drug treatment for fracture prevention. We also compared observed and predicted 10 year fracture probabilities within distinct groups identified by osteoporosis status and fracture risk category.

\section{Methods \\ Study population}

From 2000 through 2002, 5994 men who were aged at least 65 years were recruited for participation in the baseline examination of the Osteoporotic Fractures in Men (MrOS) study. ${ }^{30}{ }^{31}$ The enrollment criteria were designed to recruit a cohort that represented the characteristics of community dwelling older men residing in the United States. Men were recruited from population based listings in six regions (Birmingham, Alabama; the Monongahela Valley near Pittsburgh, Pennsylvania; Minneapolis, Minnesota; Palo Alto, California; San Diego, California; and Portland, Oregon). Men with a history of bilateral hip replacement, men who were unable to walk without the assistance of another person, and men with a medical condition (in the judgment of the investigator) that would result in imminent death were excluded. The cohort for this analysis comprised the 5880 men not taking bisphosphonates at the baseline examination with hip and spine bone mineral density measurements, assessment of FRAX clinical risk factors, and incident fracture ascertainment during 10 years of follow-up.

\section{Clinical risk factors and bone mineral density}

Participants were asked at the baseline examination about race/ethnicity, history of fracture since the age of 50, diagnosis by a physician of rheumatoid arthritis, parental history of hip fracture, smoking status, and alcohol intake. Participants were asked to bring all drug containers used within the preceding 30 days with them to the clinic visit. Drugs were identified and recorded by the clinic staff, and the information was stored in an electronic drugs inventory database. ${ }^{32}$

Measurements of body weight and height were used to calculate a standard body mass index. Bone mineral density of the lumbar spine and total hip including the femoral neck subregion was measured using dual energy x ray absorptiometry (QDR 4500W, Hologic, Waltham, MA). ${ }^{33}$ As recommended by WHO, ${ }^{6}$ female specific $\mathrm{T}$ scores at the femoral neck were calculated on the 
basis of the mean and standard deviation obtained from the third National Health and Nutrition Examination Survey (NHANES III) white female reference population aged $20-29$ years. ${ }^{34}{ }^{35} \mathrm{~A}$ female specific $\mathrm{T}$ score at the femoral neck of -2.5 or below corresponds to an absolute value of $0.558 \mathrm{~g} / \mathrm{cm}^{2}$ or lower. As recommended by US professional societies including the $\mathrm{NOF}^{7-9}{ }^{36}$ male specific $\mathrm{T}$ scores at the femoral neck and total hip were calculated on the basis of means and standard deviations obtained from the NHANES III white male reference population aged 20-29 years, ${ }^{35}$ and male specific $\mathrm{T}$ scores at the lumbar spine were calculated on the basis of the mean and standard deviation obtained from the densitometer manufacturer's reference database for 30 year old white men. ${ }^{37}$ A male specific $\mathrm{T}$ score of -2.5 or below, as referenced in the US treatment guidelines, corresponds to an absolute value of $0.592 \mathrm{~g} / \mathrm{cm}^{2}$ or lower at the femoral neck, $0.681 \mathrm{~g} / \mathrm{cm}^{2}$ or lower at the total hip, or $0.816 \mathrm{~g} / \mathrm{cm}^{2}$ or lower at the total lumbar spine (lumbar vertebra 1-4).

Information on bisphosphonate use (any use within the previous 30 days) was also collected during the 10 year follow-up period at the following time points: first interim questionnaire (mean 2.0 (SD 0.2) years after baseline examination), first sleep examination (3.4 (0.5) years), second examination (4.6 (0.4) years), third examination (6.9 (0.4) years), second interim questionnaire (8.9 (0.4) years), and second sleep examination (9.9 (0.8) years).

\section{FRAX tool and 10 year predicted fracture probabilities}

The FRAX web based tool is publicly available and can be used for free by providers and patients. For example, when a provider sees a patient, the provider or a designated staff member can enter the FRAX clinical risk factor values (components) into the web based tool, which instantly calculates the 10 year predicted probabilities of hip fracture and major osteoporotic fracture (defined by FRAX as hip, clinical vertebral, wrist, or proximal humerus fracture). However, the specific equations and algorithms developed by the WHO Collaborating Centre for Metabolic Bone Disease, University of Sheffield, UK that generate predicted FRAX fracture probabilities to make calculation of the FRAX probabilities for all individuals in a large population feasible have not been published. Thus, to calculate the US FRAX 10 year predicted probabilities of hip and major osteoporotic fracture for all 5800 men in this study, we transmitted data for each participant on FRAX clinical risk factors (age, sex, race/ethnicity, body mass index, history of fracture, parental history of hip fracture, smoking and alcohol consumption, use of oral glucocorticoids, and history of rheumatoid arthritis, and femoral neck bone mineral density data (absolute value in $\left.\mathrm{g} / \mathrm{cm}^{2}\right)$ ) in a confidential and secure manner to the WHO center, where the probabilities were computed using FRAX models with bone mineral density (version 3.3).

If data were missing on any of the clinical characteristics (other than age, race/ethnicity, body mass index, and bone mineral density, for which none of the 5880 men had missing data), we used the default (no) value, as is recommended by the WHO center. ${ }^{21}$ For example, if smoking status was missing for a participant, we classified the participant as a non-smoker. Data were $100 \%$ complete for all characteristics for 4285 men $(73 \%$ of the cohort). By far the most common characteristic for which data were missing was "parental history of hip fracture," for which 1405 (24\%) of men reported "don't know." Information on drugs used for the definition of "oral glucocorticoid use" was missing for 238 (4\%) men. Other risk factors had missing data for nine or fewer men.

\section{Ascertainment of fractures}

After the baseline examination, we contacted surviving participants every four months about fractures during the subsequent 10 year follow-up period. We selected a 10 year time point to match that specified by the FRAX tool. More than $99 \%$ of these follow-up contacts were completed. We confirmed fractures by review of radiographic reports. Incident fracture outcomes for this analysis included hip fracture and major osteoporotic fracture. Follow-up time for the analysis of a given fracture outcome ended at the date of the first fracture event, at the date of death, at the date of termination of participation in the MrOS study, or after 10 years of follow-up. Mean follow-up for the cohort was 8.6 (SD 2.5) years for hip fracture and 8.5 (2.6) years for major osteoporotic fracture. Of the 5880 men included in this study, 1634 (27.9\%) died and 214 (3.6\%) terminated before reaching 10 years of follow-up.

\section{Statistical analysis}

To clearly illustrate the incremental effect of both broadening the definition of osteoporosis and use of FRAX intervention thresholds on the proportion of the male population identified as candidates for treatment, we sequentially applied the WHO osteoporosis definition, the NOF osteoporosis definition, and NOF derived FRAX intervention thresholds to the 5880 men, resulting in four mutually exclusive groups: osteoporosis by WHO criteria alone; osteoporosis by NOF (but not WHO) criteria; no osteoporosis but at high risk of fracture (that is, male specific $\mathrm{T}$ score at femoral neck, lumbar spine, and total hip above -2.5 and predicted 10 year probability of hip fracture at least $3 \%$ or predicted 10 year probability of major osteoporotic fracture at least 20\%); and no osteoporosis and at low risk of fracture (that is, male specific T score at femoral neck, lumbar spine, and total hip above -2.5 , predicted 10 year probability of hip fracture less than $3 \%$, and predicted 10 year probability of major osteoporotic fracture less than $20 \%$ ).

We calculated observed 10 year probabilities of confirmed incident hip and major osteoporotic fracture events for each of the four groups by using cumulative incidence estimation, accounting for the competing risk of mortality. ${ }^{38}$ We used FRAX models with femoral neck bone mineral density to estimate predicted 10 year probabilities for these two outcomes for each of the four groups.

In the primary analysis for calculating observed fracture probabilities, we retained men starting bisphosphonate treatment during the follow-up period in the cohort because censoring them at the time of starting treatment might lead to bias in the comparison of the observed and predicted fracture probabilities. To determine the effect of alternative approaches for handling treatment initiation, we did two sensitivity analyses. The first calculated observed fracture probabilities with censoring of men starting bisphosphonates during follow-up at the estimated time of initiation, and the second calculated observed fracture probabilities with treatment initiation considered as a competing risk. For the 426 men who started bisphosphonates during follow-up, the estimated time of initiation for a given participant was the midpoint between the last known time point when he was a non-user and the first time point when bisphosphonate use was recorded.

As the NOF guideline specifically recommends that drug treatment should be considered in men with low bone mineral density but not osteoporosis who are at or above the NOF 
derived intervention threshold, we also did a secondary analysis limiting the "no osteoporosis but at high fracture risk" group $(n=936)$ to the 859 men with a male specific bone mineral density $\mathrm{T}$ score between -1.01 and -2.49 and predicted 10 year probability of hip fracture of at least $3 \%$ or predicted 10 year probability of major osteoporotic fracture of at least $20 \%$. Finally, we did sensitivity analyses limiting the cohort to the 4285 men with complete data on all clinical characteristics used in the calculation of FRAX 10 year fracture probabilities.

We used SAS version 9.2 for all analyses except those using cumulative incidence estimation, for which we used Stata version 12.1 .

\section{Results}

Among the cohort of 5880 men, 130 (2.2\%) were identified as having osteoporosis by using WHO criteria based on a female specific $\mathrm{T}$ score of -2.5 or below at the femoral neck. With subsequent application of the NOF definition, an additional 422 men were identified as having osteoporosis, resulting in a total prevalence of osteoporosis (based on male specific T score of -2.5 or below at the femoral neck, total hip, or lumbar spine) of $9.4 \%$. Of these 422 men who met NOF (but not WHO) criteria for osteoporosis, 142 men had a male specific bone mineral density $\mathrm{T}$ score of -2.5 or below at the femoral neck ( 15 additional men were then subsequently identified owing to a male specific bone mineral density $\mathrm{T}$ score of -2.5 or below at the total hip, and finally 265 additional men were subsequently identified owing to a male specific $\mathrm{T}$ score of -2.5 or below at the lumbar spine). With subsequent application of the FRAX intervention thresholds proposed by the NOF, 936 (15.9\%) additional men without osteoporosis by any criteria were identified as being at high risk of fracture, raising the total prevalence of men potentially eligible for drug treatment to $25.3 \%$. Of these 936 men, $859(91.8 \%)$ had a male specific bone mineral density $\mathrm{T}$ score between -1.01 and -2.49 .

The mean age of men in the overall cohort was 73.6 (range 65-100) years. The table $\downarrow$ shows the characteristics of the cohort according to category of osteoporosis definition and fracture risk. In particular, among the 936 men without osteoporosis by WHO or NOF bone mineral density criteria but who were classified as being at high risk of fracture by virtue of their FRAX 10 year predicted fracture probabilities, the mean age and proportion with previous fracture were similar to those among the 130 men with osteoporosis by WHO criteria. Among this "no osteoporosis but at high fracture risk" group, 934 $(99.8 \%)$ men had a FRAX 10 year probability of hip fracture of $3 \%$ or higher, but only $65(6.9 \%)$ had a 10 year probability of major osteoporotic fracture of $20 \%$ or higher (63 men met both FRAX intervention thresholds, 871 men were identified on the basis of the $3 \%$ hip threshold alone, and two men were identified on the basis of the $20 \%$ major osteoporotic fracture threshold alone). Among the 130 men with osteoporosis by WHO criteria, $128(98.5 \%)$ had a 10 year probability of hip fracture of $3 \%$ or higher and $30(23.1 \%)$ had a 10 year probability of major osteoporotic fracture of $20 \%$ or higher $(30$ men met both FRAX intervention thresholds, 98 were identified on the basis of the $3 \%$ hip threshold alone, none was identified on the basis of the $20 \%$ major osteoporotic fracture threshold alone, and two met neither FRAX intervention threshold).

During the 10 year follow-up period, 177 (3.0\%) men had a hip fracture. Observed hip fracture probabilities incrementally decreased across the groups defined by category of osteoporosis definition and fracture risk, ranging from $20.6 \%$ (osteoporosis by WHO criteria) to $6.8 \%$ (osteoporosis by NOF (but not WHO) criteria), $6.4 \%$ (no osteoporosis but at high fracture risk), and $1.5 \%$ (no osteoporosis and at low fracture risk) (fig $1 \Downarrow$ ). Among men with osteoporosis, the observed hip fracture probabilities were greater than the predicted probabilities. In men with osteoporosis by WHO criteria, the observed probability was $20.6 \%$ (95\% confidence interval $14.0 \%$ to $28.0 \%$ ) compared with the FRAX predicted probability of $9.5 \%$ (8.2\% to $10.7 \%$ ). On the other hand, the observed and predicted hip fracture probabilities were similar among men without osteoporosis, including those at high and those at low fracture risk.

A total of $429(7.3 \%)$ men had a major osteoporotic fracture during the 10 year follow-up period. Observed fracture probabilities ranged from $30.0 \%$ (osteoporosis by WHO criteria) to $18.0 \%$ (osteoporosis by NOF (but not WHO) criteria), $12.0 \%$ (no osteoporosis but at high fracture risk), and $4.8 \%$ (no osteoporosis and at low fracture risk) (fig $2 \Downarrow$ ). Similar to the results for hip fracture, observed probabilities of major osteoporotic fracture were greater than predicted probabilities among men with osteoporosis, especially those identified by WHO criteria for whom the observed probability was $30.0 \%$ ( $22.2 \%$ to $38.1 \%$ ) compared with the FRAX predicted probability of $17.4 \%$ (16.0\% to $18.8 \%)$. This discrepancy was again not apparent among men without osteoporosis, including those at high and those at low fracture risk.

Findings for the comparisons of observed to predicted fracture probabilities were similar in analyses in which men starting bisphosphonates during follow-up were censored at the time of initiation or when initiation of bisphosphonates was considered as a competing risk. In addition, results were not altered in analyses in which the group of men without osteoporosis but at high fracture risk was limited to the 859 men with male specific bone mineral density $\mathrm{T}$ scores between -1.01 and -2.49 . Finally, limiting the cohort to the 4285 men with complete data on all clinical characteristics used in the calculation of FRAX 10 year fracture probabilities did not alter our findings for the proportion of men identified as candidates for drug treatment or results on observed and predicted fracture probabilities.

\section{Discussion}

In this cohort of community dwelling older men living in the United States, we found that the choice of definition of osteoporosis and use of National Osteoporosis Foundation derived FRAX intervention thresholds had major effects on the proportion of men identified as candidates for drug treatment to prevent fracture. Expanding the indication for treatment from men with osteoporosis defined by WHO criteria to men meeting criteria using FRAX intervention thresholds proposed by the NOF resulted in more than a 10 -fold rise in the proportion of men identified as candidates for drug treatment. Among the $2 \%$ of men identified as having osteoporosis by WHO criteria, who constitute the group most likely to benefit from drug treatment to prevent fracture, observed 10 year fracture probabilities were highest and exceeded FRAX predicted probabilities.

\section{Comparison with other studies}

Choice of normative database and number of skeletal sites considered had a substantial effect on the estimated prevalence of osteoporosis in older men in this study; a fourfold increase in the prevalence occurred when we defined osteoporosis by using NOF (male normative database, three skeletal sites) instead of WHO (female normative database, one skeletal site) criteria. These findings are in agreement with most, ${ }^{12} 3539$ but not all, ${ }^{11}$ previous studies. Use of a female derived bone mineral density T score threshold to define osteoporosis in men is 
supported by a meta-analysis that reported similar relative risks of fracture per unit decrease in femoral neck bone mineral density in men and women and similar age adjusted hip fracture rates in men and women for any given absolute value of femoral neck bone mineral density. ${ }^{41}$ Both the NOF and the Endocrine Society in the United States recommend use of male derived bone mineral density $\mathrm{T}$ score thresholds. ${ }^{7-9}$ In contrast, the International Society of Clinical Densitometry recently reversed its earlier position advocating use of a young male normative database and recommended that $\mathrm{T}$ scores in men be calculated using a young female normative database..$^{10} 3642$

Nearly one out of every six older men in this cohort did not meet criteria for osteoporosis using even the most liberal definition but were classified as at high risk of fracture on the basis of having a 10 year probability of hip or major osteoporotic fracture at or above FRAX intervention thresholds proposed by the NOF. Of these, the vast majority (92\%) had male specific bone mineral density $\mathrm{T}$ score between -1.01 and -2.49 and would be candidates for drug treatment according to guidelines proposed by the NOF and endorsed by other US professional societies. ${ }^{7-9} 36$ Our finding that the application of NOF treatment guidelines markedly increased the proportion of older men identified as candidates for treatment is consistent with those reported in other studies, ${ }^{43}$ including a previous analysis of this cohort. ${ }^{44}$ Our results expand on these previous cross sectional reports by clearly showing the incremental effect of broadening the definition of osteoporosis and use of the FRAX intervention thresholds recommended by the NOF.

Although basing the treatment decision on a patient's absolute risk of fracture is appealing, the efficacy of currently available treatment in preventing clinical fractures in men and women without osteoporosis defined by female specific norms or existing vertebral fractures has not been demonstrated. Previous trials in men with low bone mineral density have been powered to detect a change in bone mineral density or a reduction in risk of new vertebral fractures identified on the basis of radiographs alone ${ }^{13-17}$; no trials in men have shown a benefit of treatment in reducing the risk of clinical fractures. With the exception of one trial in patients with recent hip fracture, ${ }^{19}$ evidence of the efficacy of treatment in reducing clinical fractures is limited to trials in postmenopausal women with osteoporosis defined by a female specific bone mineral density $\mathrm{T}$ score of -2.5 or below or with existing radiographic vertebral fractures. ${ }^{18}$ Systematic reviews of trials of bisphosphonates in postmenopausal women have not reported significant reductions in risk of non-vertebral fractures among women without osteoporosis or existing vertebral fractures. ${ }^{18}{ }^{45}$ Although one trial of clodronate reported efficacy of treatment in reducing clinical fractures (but not hip fractures) in older women not selected on the basis of bone mineral density, ${ }^{46}$ other pivotal trials of alendronate, risedronate, and denosumab found no benefit of treatment in reducing clinical fractures among women not selected on the basis of the WHO definition of osteoporosis (that is, female specific bone mineral density $\mathrm{T}$ score -2.5 or below) or existing vertebral fractures. ${ }^{47-49}$ In addition, whether higher estimated FRAX probabilities identify a subgroup of patients who benefit more from treatment is unknown. Post hoc analyses of trials in women of drugs including raloxifene and alendronate have not found evidence of an interaction between level of FRAX 10 year fracture probability and treatment assignment for prediction of risk of fracture events, ${ }^{50}{ }^{51}$ although some evidence supported an interaction in trials of denosumab and clodronate. ${ }^{52} 53$

Our results indicate that using criteria other than the WHO definition of osteoporosis to select older men for drug treatment substantially increases the pool of men labeled as abnormal, with potential consequences of overdiagnosis and overtreatment. Because considerable uncertainty exists, randomized trials are clearly warranted to evaluate the efficacy of treatment in reducing clinical fractures in older adults without osteoporosis but at high fracture risk before expanding indications for drug treatment.

The performance of the FRAX tool has been the subject of multiple previous studies, including a recent study using data collected in the Osteoporotic Fractures in Men (MrOS) study that evaluated the performance of FRAX models with and without bone mineral density in the overall cohort. ${ }^{54}$ Findings suggested that discrimination of men with and without incident fracture events was improved by addition of bone mineral density to the calculation, although even the models with bone mineral density were limited in their ability to predict fracture events, especially non-hip fractures (the area under the curve statistic for hip fracture was 0.67 without and 0.77 with bone mineral density; for major osteoporotic fracture it was 0.63 without and 0.67 with bone mineral density). Results also indicated suboptimal calibration of FRAX models with bone mineral density (underestimation of hip fracture probability and overestimation of major osteoporotic fracture probability). The observed 10 year cumulative incidence hip fracture probability was $3.0 \%$ compared with a mean 10 year FRAX predicted probability of $2.3 \%$, and the observed 10 year cumulative incidence major osteoporotic fracture probability was $6.9 \%$ compared with a mean 10 year FRAX predicted probability of $7.6 \% .^{54}$

Our study determined observed and FRAX predicted probabilities within mutually exclusive groups of men identified by osteoporosis status and fracture risk, as US professional societies have strongly recommended that clinicians consider both bone mineral density and fracture risk defined using NOF derived FRAX intervention thresholds in making the critical decision about whether to recommend drug treatment to a patient. We found a wide range of observed 10 year probabilities of hip and major osteoporotic fracture events across these distinct groups of men. As expected, observed probabilities were highest among men with osteoporosis as defined by WHO criteria using a young female specific normative database, intermediate among men with osteoporosis defined by NOF (but not WHO) criteria using a male specific normative database, intermediate among men without osteoporosis but at high fracture risk, and lowest among men without osteoporosis and at low fracture risk. Among men with osteoporosis by WHO criteria, one out of every five had a hip fracture and three out of every 10 had a major osteoporotic fracture. On the basis of evidence from randomized trials in women, these men with osteoporosis defined using a young female specific normative database are most likely to benefit from drug treatment to prevent fracture. However, our results indicated that fracture probabilities as calculated by the FRAX tool in this group of men underestimated their actual fracture risk. Because the FRAX tool did not underestimate fracture risk among men without osteoporosis, differences in predicted fracture probabilities across groups were smaller than actual observed differences in fracture probabilities. As patients may be prepared accept a higher absolute fracture risk than providers are before considering drug treatment to be justified, ${ }^{55}$ information about absolute fracture risk must be accurately conveyed to the patients most likely to benefit from treatment. In addition, evidence suggests that a large discrepancy may exist between NOF recommended FRAX intervention thresholds and acceptable intervention thresholds of patients. ${ }^{55}$ 
In addition to FRAX, many other tools for assessing fracture risk have been developed. A recent systematic review of these tools identified a total of 48 instruments, ${ }^{56}$ but only six were validated more than once in a population based setting with acceptable methodological quality. None of these six tools consistently performed better than the others, and simple tools often did as well as or better than more complex tools including FRAX. No studies determined the effectiveness of tools in selecting patients for treatment or reported that a screening and/or treatment strategy based on a specific tool reduced the risk of clinical fractures. Thus, randomized trials are needed to evaluate the comparative effectiveness of strategies based on fracture risk assessment tools in reducing risk of clinical fracture events before widespread adoption of these tools into clinical decision making in the practice setting regarding whether to recommend drug treatment. In addition, decreasing the societal burden of fractures among older adults will require effective interventions beyond available skeletal treatments targeted at patients with low bone mineral density. Thus, randomized trials are also warranted to evaluate the efficacy of non-skeletal interventions (for example, community based fall prevention strategies) in reducing fracture events.

\section{Strengths and limitations of study}

This study has several strengths, including the large, well characterized cohort with nearly complete long term follow-up of fracture events. Observed fracture probabilities were adjusted for the competing risk of mortality.

However, this study has several limitations. The cohort comprised community dwelling, predominantly white men living in the United States. Characteristics including status of clinical risk factors for fracture and hip bone mineral density were in general similar to those of a population based sample of men aged 65 years and over enrolled in NHANES III, ${ }^{44}$ but fewer men in the MrOS study were current smokers. This may in part explain the discrepancy between observed and predicted FRAX fracture probabilities among men with osteoporosis in our cohort, as smoking is a risk factor for mortality.

Our findings on the effect of using FRAX intervention thresholds on the proportion of men identified as warranting drug treatment are limited to the thresholds identified by the NOF for the United States and do not necessarily apply to strategies proposed in other countries. ${ }^{57-59}$ For example, the National Osteoporosis Guideline Group ${ }^{58}$ in collaboration with other societies in the United Kingdom, recommends using the FRAX 10 year probability of major osteoporotic fracture calculated without bone mineral density to assess the need for bone mineral density testing in an adults aged 50 years and older; among patients requiring a bone mineral density test, the re-calculated FRAX 10 year probability of major osteoporotic fracture with bone mineral density is used to determine whether drug treatment is warranted. In these guidelines, FRAX intervention thresholds for bone mineral density testing and for starting drug treatment vary according to age. To our knowledge, the implications of using these guidelines on the proportion of UK adults identified as candidates for drug treatment to prevent fracture have not been examined. Our findings suggest that use of a single FRAX intervention threshold based on probability of major osteoporotic fracture of $20 \%$ would identify a much smaller proportion of older men without evidence of osteoporosis (by any definition) as warranting drug treatment, as the vast majority of men in this group in our study were identified as being eligible for treatment because they met or exceeded the hip fracture threshold of $3 \%$. However, our findings also indicate that reliance on the FRAX $20 \%$ threshold alone without consideration of bone mineral density would fail to identify more than $75 \%$ of men with osteoporosis by WHO criteria.

Although evidence exists showing the efficacy of several drug treatments in preventing clinical fractures among postmenopausal women with existing vertebral fractures, ${ }^{60}$ and one randomized trial has shown efficacy of zoledronic acid in preventing symptomatic fractures among patients with recent hip fracture, ${ }^{19}$ our study did not specifically look at the effect of selecting older men for drug treatment on the basis of these criteria. Because the equations and algorithms that generate the fracture risk probabilities have not been published for the FRAX tool, this study could not investigate the relative importance of individual FRAX clinical risk factors in fracture prediction. Finally, the absolute number of fracture events in each of the four groups of men in this study was small, and our findings should be confirmed using data collected in other long term prospective studies of older men.

\section{Conclusions and implications}

In conclusion, broadening the indication for drug treatment beyond men with osteoporosis as defined by WHO criteria resulted in a large increase in the proportion of older community dwelling men in the United States who were identified as warranting drug treatment to prevent fracture. Men with osteoporosis by WHO criteria had observed probabilities of clinical fracture events that exceeded their FRAX predicted probabilities. Based on data from trials in women, these men with osteoporosis by WHO criteria are most likely to benefit from treatment. As the goal of treatment is to prevent clinical (symptomatic) fractures, our results in the context of the available medical literature indicate that treatment should be offered to men who meet WHO criteria for osteoporosis (that is, femoral neck bone mineral density of $0.558 \mathrm{~g} / \mathrm{cm}^{2}$ or below), men with existing vertebral fractures, and men with a recent history of hip fracture. Expanding indications for treatment beyond this group has unknown value owing to lower observed fracture probabilities and uncertain benefits of treatment and would result in overdiagnosis and overtreatment. Randomized clinical trials are warranted to evaluate the efficacy of drug treatment in reducing clinical fractures in older men and women without osteoporosis but at high risk of fracture before a fundamental change in the strategy used to identify candidates for drug treatment is adopted.

Contributors: KEE and ESO were involved in the design and conduct of the study and the collection of data. All authors were involved in the analysis and/or interpretation of data. KWP did the statistical analyses; she had full access to all the data in the study and takes responsibility for the integrity of the data and the accuracy of the data analyses. KEE was responsible for preparation of the manuscript. KWP, BCT, MLG, MGD, WDL, TLB, HAF, and ESO critically reviewed and approved the manuscript. KEE and ESO are the guarantors.

Funding: The Osteoporotic Fractures in Men (MrOS) Study is supported by National Institutes of Health $(\mathrm{NIH})$ funding. The following institutes provide support: the National Institute of Arthritis and Musculoskeletal and Skin Diseases, the National Institute on Aging, the National Center for Research Resources, and NIH Roadmap for Medical Research under the following grant numbers: U01 AR45580, U01 AR45614, U01 AR45632, U01 AR45647, U01 AR45654, U01 AR45583, U01 AG18197, U01 AG027810, and UL1 TR000128. The funding agencies had no direct role in the conduct of the study; the collection, management, analyses, and interpretation of the data; or the preparation or approval of the manuscript. 


\section{What is already known on this topic}

The efficacy of drug treatment in preventing clinical (symptomatic) fractures in men is unknown, but treatment in postmenopausal women with osteoporosis reduces the risk of clinical fractures

Several US professional societies now recommend using cut points of 10 year absolute fracture risk as estimated by the FRAX tool in making decisions about whether to start drug treatment in adults aged 50 or over

Previous studies have suggested that application of these recommended FRAX intervention thresholds increases the proportion of older adults identified as candidates for drug treatment

However, the incremental impact of both broadening the definition of osteoporosis and use of FRAX intervention thresholds on the proportion of older men identified as candidates for drug treatment is uncertain

\section{What this study adds}

Depending on the definition of osteoporosis and the FRAX absolute fracture risk cut points applied to the study population, the proportion of men labeled as abnormal and warranting treatment varied from $2 \%$ up to $25 \%$

Among men identified as having osteoporosis according to a stringent definition, who comprised $2 \%$ of the study population, actual observed fracture probabilities during 10 years of follow-up were highest and exceeded FRAX predicted fracture probabilities Based on results of randomized trials in women, these men are most likely to benefit from drug treatment to prevent fracture

Competing interests: All authors have completed the ICMJE uniform disclosure form at www.icmje.org/coi_disclosure.pdf (available on request from the corresponding author) and declare: KEE serves as a consultant on a data monitoring committee for Merck Sharpe \& Dohme; WDL has served as a speaker for Amgen, Eli Lilly, and Novartis and received research grants from Novartis, Amgen, and Genzyme; ESO receives research support from Merck Sharpe \& Dohme and Eli Lilly and provides consultation to Amgen, Merck Sharpe \& Dohme, and Eli Lilly; no other relationships or activities that could appear to have influenced the submitted work.

Ethical approval: The institutional review board at each participating institution approved the study protocol, and all participants gave informed consent.

Transparency statement: The first author (KEE) affirms that this manuscript is an honest, accurate, and transparent account of the study being reported; that no important aspects of the study have been omitted; and that any discrepancies from the study as planned (and, if relevant, registered) have been explained.

Data sharing: The Osteoporotic Fractures in Men (MrOS) technical appendix, statistical code, and dataset are maintained at the Data Coordinating Center (California Pacific Medical Center Research Institute, San Francisco, CA, USA) and are available from Kathy Peters (KPeters@sfcc-cpmc.net).

1 Burge R, Dawson-Hughes B, Solomon DH, Wong JB, King A, Tosteson A. Incidence and economic burden of osteoporosis-related fractures in the United States, 2005-2025. $J$ Bone Miner Res 2007;22:465-75.

2 Johnell O, Kanis J. Epidemiology of osteoporotic fractures. Osteoporos Int 2005;16(suppl 2):S3-7.

3 Stone KL, Seeley DG, Lui LY, Cauley JA, Ensrud K, Browner WS, et al. BMD at multiple sites and risk of fracture of multiple types: long-term results from the Study of Osteoporotic Fractures. J Bone Miner Res 2003;18:1947-54.

$4 \quad$ Ebeling PR. Osteoporosis in men. N Engl J Med 2008;358:1474-82.

5 Assessment of fracture risk and its application to screening for postmenopausal osteoporosis: report of a WHO study group. World Health Organ Tech Rep Ser 1994;843:1-129.

6 Kanis JA, McCloskey EV, Johansson H, Oden A, Melton LJ III, Khaltaev N. A reference standard for the description of osteoporosis. Bone 2008;42:467-75

7 National Osteoporosis Foundation. Clinician's guide to prevention and treatment of osteoporosis. NOF, 2010 (available at www.nof.org/files/nof/public/content/file/344/upload/ 159.pdf).

8 National Osteoporosis Foundation. Clinician's guide to prevention and treatment of osteoporosis. NOF, 2014 (available at http://nof.org/files/nof/public/content/file/2791/ upload/919.pdf)

9 Watts NB, Adler RA, Bilezikian JP, Drake MT, Eastell R, Orwoll ES, et al. Osteoporosis in men: an Endocrine Society clinical practice guideline. J Clin Endocrinol Metab 2012:97:1802-22

10 International Society for Clinical Densitometry. 2013 ISCD official positions-adult. www. iscd.org/official-positions/2013-iscd-official-positions-adult/.

11 Kanis JA, Bianchi G, Bilezikian JP, Kaufman JM, Khosla S, Orwoll E, et al. Towards a diagnostic and therapeutic consensus in male osteoporosis. Osteoporos Int 2011;22:2789-98

12 Richy F, Gourlay ML, Garrett J, Hanson L, Reginster JY. Osteoporosis prevalence in men varies by the normative reference. J Clin Densitom 2004;7:127-33.

13 Boonen S, Orwoll ES, Wenderoth D, Stoner KJ, Eusebio R, Delmas PD. Once-weekly risedronate in men with osteoporosis: results of a 2-year, placebo-controlled, double-blind, multicenter study. J Bone Miner Res 2009;24:719-25.

14 Boonen S, Reginster JY, Kaufman JM, Lippuner K, Zanchetta J, Langdahl B, et al. Fracture risk and zoledronic acid therapy in men with osteoporosis. N Engl J Med 2012;367:1714-23.
15 Orwoll E, Ettinger M, Weiss S, Miller P, Kendler D, Graham J, et al. Alendronate for the treatment of osteoporosis in men. N Engl J Med 2000;343:604-10.

16 Orwoll E, Teglbjaerg CS, Langdahl BL, Chapurlat R, Czerwinski E, Kendler DL, et al. A randomized, placebo-controlled study of the effects of denosumab for the treatment of men with low bone mineral density. J Clin Endocrinol Metab 2012;97:3161-9.

17 Orwoll ES, Scheele WH, Paul S, Adami S, Syversen U, Diez-Perez A, et al. The effect of teriparatide [human parathyroid hormone (1-34)] therapy on bone density in men with osteoporosis. J Bone Miner Res 2003;18:9-17.

18 MacLean C, Newberry S, Maglione M, McMahon M, Ranganath V, Suttorp M, et al. Systematic review: comparative effectiveness of treatments to prevent fractures in men and women with low bone density or osteoporosis. Ann Intern Med 2008;148:197-213.

19 Lyles KW, Colon-Emeric CS, Magaziner JS, Adachi JD, Pieper CF, Mautalen C et al. Zoledronic acid and clinical fractures and mortality after hip fracture. N Engl J Med 2007;357:1799-809.

20 Kanis JA. Diagnosis of osteoporosis and assessment of fracture risk. Lancet 2002;359:1929-36.

21 FRAX WHO Fracture Risk Assessment Tool. 2011. www.shef.ac.uk/FRAX

22 Kanis JA on behalf of the World Health Organization Scientific Group. Assessment of osteoporosis at the primary health-care level. WHO Collaborating Centre for Metabolic Bone Diseases, University of Sheffield, 2007 (available at www.shef.ac.uk/FRAX/pdfs/ WHO_Technical_Report.pdf).

23 Kanis JA, Oden A, Johansson H, Borgstrom F, Strom O, McCloskey E. FRAX and its applications to clinical practice. Bone 2009;44:734-43.

24 Hans DB, Kanis JA, Baim S, Bilezikian JP, Binkley N, Cauley JA, et al. Joint officia positions of the International Society for Clinical Densitometry and International Osteoporosis Foundation on FRAX(R): executive summary of the 2010 Position Development Conference on Interpretation and use of $\operatorname{FRAX}(\mathrm{R})$ in clinical practice. $J$ Clin Densitom 2011;14:171-80.

25 Endocrine Society. The Endocrine Society's statement on industry relationships. 2009 www.endocrine.org/ /media/endosociety/Files/About\%20Us/Corporate\%20Info/TheEndocrine-Society-s-Statement-on-Industry-Relationships-Final-11-13-09.pdf.

26 International Osteoporosis Foundation. Annual report 2012. IOF, 2013 (available at www. iofbonehealth.org/sites/default/files/PDFs/2012-IOF_Annual_Report\%5BWEB\%5D_0. pdf).

27 National Osteoporosis Foundation. 2012 annual report. NOF, 2013 (available at http:// nof.org/files/nof/public/content/report/1820/file/851.pdf).

28 Kanis JA, Johnell O, Oden A, Johansson $\mathrm{H}$, McCloskey E. FRAX and the assessment of fracture probability in men and women from the UK. Osteoporos Int 2008;19:385-97.

29 Tosteson AN, Melton LJ III, Dawson-Hughes B, Baim S, Favus MJ, Khosla S, et al. Cost-effective osteoporosis treatment thresholds: the United States perspective. Osteoporos Int 2008;19:437-47.

30 Blank JB, Cawthon PM, Carrion-Petersen ML, Harper L, Johnson JP, Mitson E, et al Overview of recruitment for the osteoporotic fractures in men study (MrOS). Contemp Clin Trials 2005;26:557-68

31 Orwoll E, Blank JB, Barrett-Connor E, Cauley J, Cummings S, Ensrud K, et al. Design and baseline characteristics of the osteoporotic fractures in men (MrOS) study-a large observational study of the determinants of fracture in older men. Contemp Clin Trials 2005;26:569-85.

32 Pahor M, Chrischilles EA, Guralnik JM, Brown SL, Wallace RB, Carbonin P. Drug data coding and analysis in epidemiologic studies. Eur J Epidemiol 1994;10:405-11.

33 Cawthon PM, Ewing SK, McCulloch CE, Ensrud KE, Cauley JA, Cummings SR, et al. Loss of hip BMD in older men: the osteoporotic fractures in men (MrOS) study. J Bone Miner Res 2009;24:1728-35.

34 Looker AC, Wahner HW, Dunn WL, Calvo MS, Harris TB, Heyse SP, et al. Updated data on proximal femur bone mineral levels of US adults. Osteoporos Int 1998;8:468-89.

35 Looker AC, Orwoll ES, Johnston CC Jr, Lindsay RL, Wahner HW, Dunn WL, et al. Prevalence of low femoral bone density in older U.S. adults from NHANES III. J Bone Miner Res 1997;12:1761-8.

36 International Society for Clinical Densitometry. 2007 ISCD official positions-adult. www. iscd.org/official-positions/2007-iscd-official-positions-adult/.

37 Kelly TL. Bone mineral density reference databases for American men and women. $J$ Bone Miner Res 1990;5:S249.

38 Coviello V, Boggess M. Cumulative incidence estimation in the presence of competing risks. Stata J 2004;4:103-12.

39 Faulkner KG, Orwoll E. Implications in the use of T-scores for the diagnosis of osteoporosis in men. J Clin Densitom 2002;5:87-93.

40 Looker AC, Melton LJ III, Borrud LG, Shepherd JA. Lumbar spine bone mineral density in US adults: demographic patterns and relationship with femur neck skeletal status. Osteoporos Int 2012;23:1351-60. 
41 Johnell O, Kanis JA, Oden A, Johansson H, De LC, Delmas P, et al. Predictive value of BMD for hip and other fractures. J Bone Miner Res 2005;20:1185-94.

42 Binkley NC, Schmeer P, Wasnich RD, Lenchik L. What are the criteria by which a densitometric diagnosis of osteoporosis can be made in males and non-Caucasians? $\mathrm{J}$ Clin Densitom 2002;5(suppl):S19-27.

43 Berry SD, Kiel DP, Donaldson MG, Cummings SR, Kanis JA, Johansson H, et al. Application of the National Osteoporosis Foundation guidelines to postmenopausal women and men: the Framingham Osteoporosis Study Osteoporos Int 2010:21:53-60.

44 Donaldson MG, Cawthon PM, Lui LY, Schousboe JT, Ensrud KE, Taylor BC, et al. Estimates of the proportion of older white men who would be recommended for pharmacologic treatment by the new US National Osteoporosis Foundation guidelines. J Bone Miner Res 2010;25:1506-11

45 Wells GA, Cranney A, Peterson J, Boucher M, Shea B, Robinson V, et al. Alendronate for the primary and secondary prevention of osteoporotic fractures in postmenopausal women. Cochrane Database Syst Rev 2008;1:CD001155.

46 McCloskey EV, Beneton M, Charlesworth D, Kayan K, deTakats D, Dey A, et al. Clodronate reduces the incidence of fractures in community-dwelling elderly women unselected for osteoporosis: results of a double-blind, placebo-controlled randomized study. J Bone Miner Res 2007;22:135-41.

47 Cummings SR, Black DM, Thompson DE, Applegate WB, Barrett-Connor E, Musliner TA, et al. Effect of alendronate on risk of fracture in women with low bone density but without vertebral fractures: results from the Fracture Intervention Trial. JAMA 1998;280:2077-82.

48 McClung MR, Geusens P, Miller PD, Zippel H, Bensen WG, Roux C, et al, for the Hip Intervention Program Study Group. Effect of risedronate on the risk of hip fracture in elderly women. N Engl J Med 2001;344:333-40.

49 McClung MR, Boonen S, Torring O, Roux C, Rizzoli R, Bone HG, et al. Effect of denosumab treatment on the risk of fractures in subgroups of women with postmenopausal osteoporosis. J Bone Miner Res 2012;27:211-8.

50 Kanis JA, Johansson H, Oden A, McCloskey EV. A meta-analysis of the efficacy of raloxifene on all clinical and vertebral fractures and its dependency on FRAX. Bon 2010;47:729-35.

51 Donaldson MG, Palermo L, Ensrud KE, Hochberg MC, Schousboe JT, Cummings SR. Effect of alendronate for reducing fracture by FRAX score and femoral neck bone mineral density: the Fracture Intervention Trial. J Bone Miner Res 2012;27:1804-10.

52 McCloskey EV, Johansson H, Oden A, Austin M, Siris E, Wang A, et al. Denosumab reduces the risk of osteoporotic fractures in postmenopausal women, particularly in those with moderate to high fracture risk as assessed with FRAX. J Bone Miner Res 2012;27:1480-6.

53 McCloskey EV, Johansson H, Oden A, Vasireddy S, Kayan K, Pande K, et al. Ten-year fracture probability identifies women who will benefit from clodronate therapy-additional results from a double-blind, placebo-controlled randomised study. Osteoporos Int 2009;20:811-7.

54 Ettinger B, Ensrud KE, Blackwell T, Curtis JR, Lapidus JA, Orwoll ES. Performance of FRAX in a cohort of community-dwelling, ambulatory older men: the Osteoporotic Fractures in Men (MrOS) study. Osteoporos Int 2013;24:1185-93.

55 Douglas F, Petrie KJ, Cundy T, Horne A, Gamble G, Grey A. Differing perceptions of intervention thresholds for fracture risk: a survey of patients and doctors. Osteoporos Int 2012;23:2135-40.

56 Rubin KH, Friis-Holmberg T, Hermann AP, Abrahamsen B, Brixen K. Risk assessment tools to identify women with increased risk of osteoporotic fracture: complexity or simplicity? A systematic review. J Bone Miner Res 2013;28:1701-17.

57 Compston J, Cooper A, Cooper C, Francis R, Kanis JA, Marsh D, et al. Guidelines for the diagnosis and management of osteoporosis in postmenopausal women and men from the age of 50 years in the UK. Maturitas 2009;62:105-8.

58 Compston J, Bowring C, Cooper A, Cooper C, Davies C, Francis R, et al. Diagnosis and management of osteoporosis in postmenopausal women and older men in the UK: National Osteoporosis Guideline Group (NOGG) update 2013. Maturitas 2013;75:392-6.

59 Papaioannou A, Morin S, Cheung AM, Atkinson S, Brown JP, Feldman S, et al. 2010 clinical practice guidelines for the diagnosis and management of osteoporosis in Canada: summary. CMAJ 2010;182:1864-73.

60 Ensrud KE, Schousboe JT. Vertebral fractures. N Engl J Med 2011;364:1634-42.

\section{Accepted: 5 June 2014}

\section{Cite this as: BMJ 2014;349:g4120}

This is an Open Access article distributed in accordance with the Creative Commons Attribution Non Commercial (CC BY-NC 3.0) license, which permits others to distribute, remix, adapt, build upon this work non-commercially, and license their derivative works on different terms, provided the original work is properly cited and the use is non-commercial. See: http://creativecommons.org/licenses/by-nc/3.0/. 


\section{Table}

Table 1/ Characteristics of $\mathbf{5 8 8 0}$ participants according to definition of osteoporosis and fracture risk category ${ }^{\star}$. Values are numbers (percentages) unless stated otherwise

\begin{tabular}{|c|c|c|c|c|c|}
\hline \multirow[b]{2}{*}{ Characteristic } & \multirow[b]{2}{*}{$\begin{array}{l}\text { Overall cohort } \\
\qquad(n=5880)\end{array}$} & \multirow{2}{*}{$\begin{array}{c}\text { Osteoporosis by } \\
\text { WHO† criteria } \\
(n=130)\end{array}$} & \multirow{2}{*}{$\begin{array}{c}\text { Osteoporosis by } \\
\text { NOF } \text { (but not WHO) } \\
\text { criteria }(n=422)\end{array}$} & \multicolumn{2}{|c|}{ No osteoporosis by WHO or NOF criteria } \\
\hline & & & & $\begin{array}{l}\text { High fracture risk§ } \\
\qquad(n=936)\end{array}$ & $\begin{array}{l}\text { Low fracture risk§ } \\
\qquad(\mathrm{n}=4392)\end{array}$ \\
\hline Mean (SD) age, years & $73.6(5.9)$ & $77.1(6.4)$ & $74.3(6.2)$ & $77.9(5.0)$ & $72.5(5.5)$ \\
\hline \multicolumn{6}{|l|}{ Age group, years: } \\
\hline $65-69$ & $1747(30)$ & $19(15)$ & $116(27)$ & $42(4)$ & $1570(36)$ \\
\hline $70-74$ & $1678(29)$ & $25(19)$ & $105(25)$ & $184(20)$ & $1364(31)$ \\
\hline $75-79$ & $1419(24)$ & $37(28)$ & $112(27)$ & $339(36)$ & $931(21)$ \\
\hline$\geq 80$ & $1036(18)$ & $49(38)$ & $89(21)$ & $371(40)$ & $527(12)$ \\
\hline White ethnicity & $5257(89)$ & $118(91)$ & $377(89)$ & $908(97)$ & $3854(88)$ \\
\hline \multicolumn{6}{|l|}{$\begin{array}{l}\text { Mean (SD) bone mineral density, } \\
\mathrm{g} / \mathrm{cm}^{2} \text { : }\end{array}$} \\
\hline Lumbar spine & $1.17(0.25)$ & $0.94(0.19)$ & $0.88(0.13)$ & $1.12(0.19)$ & $1.22(0.24)$ \\
\hline Femoral neck & $0.79(0.13)$ & $0.52(0.04)$ & $0.65(0.08)$ & $0.69(0.07)$ & $0.83(0.11)$ \\
\hline Total hip & $0.96(0.14)$ & $0.68(0.08)$ & $0.80(0.09)$ & $0.87(0.09)$ & $1.00(0.12)$ \\
\hline $\begin{array}{l}\text { Mean (SD) body mass index, } \\
\mathrm{kg} / \mathrm{m}^{2}\end{array}$ & $27.4(3.8)$ & $24.6(3.2)$ & $26.1(4.3)$ & $26.2(3.1)$ & $27.9(3.8)$ \\
\hline Current smoker & $205(3)$ & $9(7)$ & $16(4)$ & $34(4)$ & $146(3)$ \\
\hline$\geq 3$ alcoholic drinks/day & $237(4)$ & $3(2)$ & $14(3)$ & $58(6)$ & $162(4)$ \\
\hline Any fracture since age 50 & $1299(22)$ & $52(40)$ & $121(29)$ & $381(41)$ & $745(17)$ \\
\hline Oral corticosteroid use & $477(8)$ & $13(10)$ & $57(14)$ & $110(12)$ & $297(7)$ \\
\hline Rheumatoid arthritis & $304(5)$ & $8(6)$ & $21(5)$ & $82(9)$ & $193(4)$ \\
\hline Parental history of hip fracture & $743(13)$ & $21(16)$ & $62(15)$ & $355(38)$ & $305(7)$ \\
\hline $\begin{array}{l}\text { FRAX } 10 \text { year probability of hip } \\
\text { fracture } \geq 3 \%\end{array}$ & $1299(22)$ & $128(98)$ & $237(56)$ & $934(\sim 100)$ & $0(0)$ \\
\hline $\begin{array}{l}\text { FRAX } 10 \text { year probability of major } \\
\text { osteoporotic fracture } \geq 20 \%\end{array}$ & $122(2)$ & $30(23)$ & $27(6)$ & $65(7)$ & $0(0)$ \\
\hline $\begin{array}{l}\text { Started bisphosphonate treatment } \\
\text { during follow-up }\end{array}$ & $426(7)$ & $40(31)$ & $134(32)$ & $91(10)$ & $161(4)$ \\
\hline Incident hip fracture & $177(3)$ & $26(20)$ & $28(7)$ & $59(6)$ & $64(1)$ \\
\hline $\begin{array}{l}\text { Incident major osteoporotic } \\
\text { fracture }\end{array}$ & $429(7)$ & $38(29)$ & $74(18)$ & $111(12)$ & $206(5)$ \\
\hline Died during follow-up & $1883(32)$ & $55(42)$ & $151(36)$ & $430(46)$ & $1247(28)$ \\
\hline
\end{tabular}

All characteristics assessed at baseline except for incident hip fracture, incident major osteoporotic fracture, and death.

*Fracture risk calculated using models with femoral neck bone mineral density (BMD).

†World Health Organization definition of osteoporosis based on use of female specific T score of -2.5 or below at femoral neck (that is, femoral neck BMD $\leq 0.558$ $\left.\mathrm{g} / \mathrm{cm}^{2}\right)$.

‡National Osteoporosis Foundation definition of osteoporosis based on use of male specific T score of -2.5 or below at femoral neck, total hip, or lumbar spine; this group (osteoporosis by NOF but not WHO criteria) includes men with femoral neck BMD 0.558-0.592 $\mathrm{g} / \mathrm{cm}^{2}$, total hip BMD $\leq 0.681 \mathrm{~g} / \mathrm{cm}^{2}$, or total lumbar spine $\mathrm{BMD} \leq 0.816 \mathrm{~g} / \mathrm{cm}^{2}$.

§High fracture risk as defined by FRAX (with BMD) 10 year probabilities of hip fracture $\geq 3 \%$ or major osteoporotic fracture $\geq 20 \%$; low fracture risk as defined by FRAX (with BMD) 10 year probabilities of hip fracture $<3 \%$ and major osteoporotic fracture $<20 \%$. 


\section{Figures}

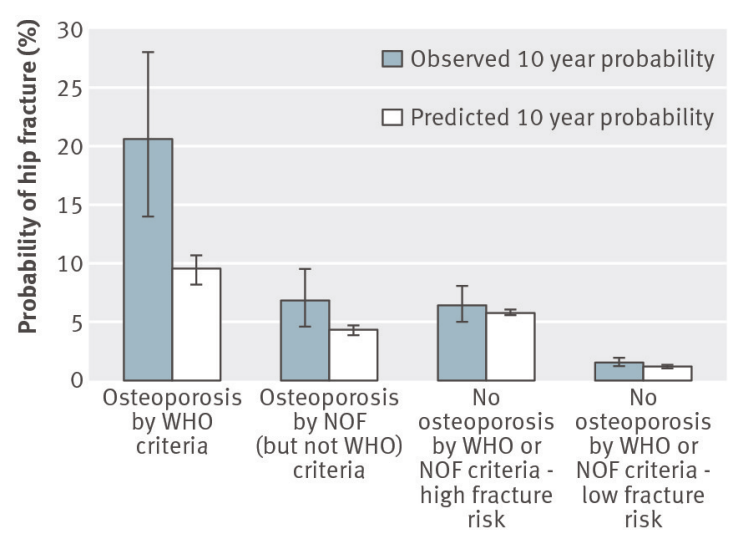

Fig 1 Observed versus predicted 10 year probability of hip fracture according to definition and fracture risk. 26 hip fractures occurred among 130 men with osteoporosis by WHO criteria alone, 28 among 422 men with osteoporosis by National Osteoporosis Foundation (NOF) (but not WHO) criteria, 59 among 936 men without osteoporosis but at high risk of fracture, and 64 among 4392 men without osteoporosis and at low risk of fracture

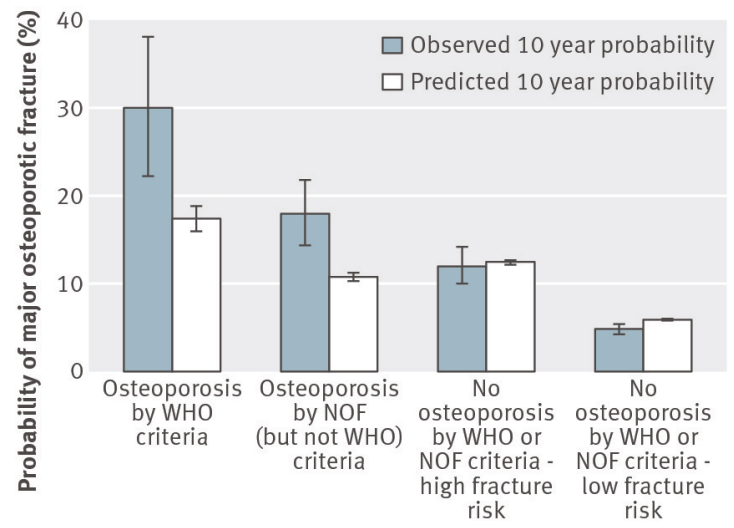

Fig 2 Observed versus predicted 10 year probability of major osteoporotic fracture according to definition and fracture risk. 38 major osteoporotic fractures occurred among 130 men with osteoporosis by WHO criteria alone, 74 among 422 men with osteoporosis by National Osteoporosis Foundation (NOF) (but not WHO) criteria, 111 among 936 men without osteoporosis but at high risk of fracture, and 206 among 4392 men without osteoporosis but at low risk of fracture 